\title{
Search for neutrinoless double-beta decay and measurement of double beta decay with two neutrinos with the NEMO-3 detector
}

John Cesar (for the NEMO-3 and SuperNEMO Collaborations) ${ }^{* \dagger}$

The University of Texas at Austin

E-mail: jpcesar@utexas.edu

\begin{abstract}
The NEMO-3 experiment operated from 2003 to 2011 with the goal of searching for neutrinoless double-beta decay. By separating the source and detector components and employing a combination of tracking and calorimetric elements, the experiment was able to capitalize on its unique approach to produce many unique physics measurements across a variety of different double-beta decay isotopes. These proceedings give the latests results from the NEMO-3 experiment including both new double-beta decay results from different isotopes and upcoming analyses beyond double-beta decay which illustrate the power and versatility of the NEMO technique.
\end{abstract}

38th International Conference on High Energy Physics

3-10 August 2016

Chicago, USA

* Speaker.

${ }^{\dagger}$ A footnote may follow. 


\section{Double-Beta Decay}

The goal of the NEMO-3 experiment is to search for the lepton number violating process known as neutrinoless double-beta decay $(0 \vee \beta \beta)$. The half-life for this process is given by,

$$
\frac{1}{T_{1 / 2}^{0 v}}=G^{0 v}\left|M^{0 v}\right|^{2}\left\langle m_{v}\right\rangle^{2}
$$

where $G^{0 v}$ is a well-known phase space factor, $M^{0 v}$ is a difficult to calculate nuclear matrix element (NME), and $\left\langle m_{v}\right\rangle$ is the effective Majorana mass term (which according to [1] arises regardless of which mechanism is behind the process). The NEMO approach to searching for this process was specifically motivated to address the two main uncertainties in this relation: obtaining the NME values and, in the event of a signal, determining the underlying physics process driving $0 v \beta \beta$ decay.

\subsection{The NEMO Technique}

The detection technique pioneered by the NEMO experiments has been that of a multi-isotope multi-observable approach. This is done by using a heterogenous detector in which the source and detector are distinct. In NEMO-3 this is achieved by forming thin source foils out of candidate isotopes and then surrounding them with tracking and calorimetry elements as shown in figure 1. This allows for identifying different particles (electrons, positrons, alphas, and gammas) and their kinematic properties.

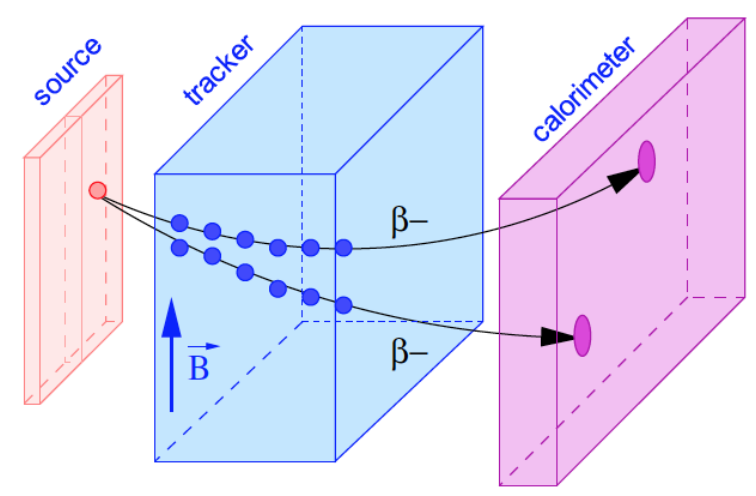

Figure 1: An illustration of the NEMO technique in which the source is made into thin modular source foils which are surrounded by a 3D tracking volume immersed in a magnetic field and a segmented calorimeter for timing and energy measurements.

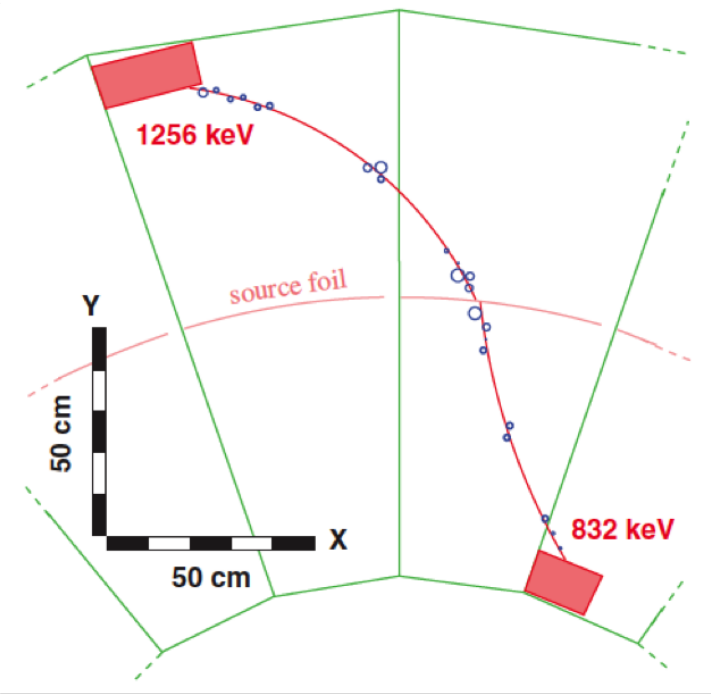

Figure 2: An illustration of a candidate double-beta decay event showing two tracks emitted from a common vertex in the source foils and with consistent curvature, each ending with separate calorimeter energy deposits.

By using this approach to make various measurements of Standard Model two-neutrino doublebeta decays $(2 v \beta \beta)$, both to the ground and excited states of various nuclei, the results can be used 
to guide nuclear models and thus NME calculations. In the event of discovery of $0 v \beta \beta$ decays, the multitude of isotopes can be used to confirm the signal while the various decay kinematics can be used to disentangle the mechanism behind the decay. As an example, NEMO-3 is uniquely able to measure the angular distribution of the emitted electrons which can be used to distinguish between the mass mechanism and right-handed current hypotheses.

\section{The NEMO-3 Experiment}

The NEMO-3 experiment employed the source + tracking + calorimetry approach in a toroidal geometry made of 20 sectors. Thin strips, $30-60 \mathrm{mg} / \mathrm{cm}^{2}$, of the source material hung vertically along the central radius. The total isotope mass, split among ${ }^{48} \mathrm{Ca},{ }^{82} \mathrm{Se},{ }^{96} \mathrm{Zr},{ }^{100} \mathrm{Mo},{ }^{16} \mathrm{Cd},{ }^{130} \mathrm{Te}$, and ${ }^{150} \mathrm{Nd}$, was about $10 \mathrm{~kg}$. The tracking volume on either side of the foils consisted of $6180 \mathrm{drift}$ wire cells operating in Geiger mode. The calorimeter's 1940 modules (plastic scintillator blocks coupled to 3" or 5" PMTs) fully covered both the inner and outer walls and partially covered the top and bottom walls. The detector was also surrounded by a solenoid producing a 25 Gauss magnetic field (for charge reconstruction), shielding in the form of wood, iron, and borated water for the external gamma and neutron flux, and an anti-radon tent to suppress the presence of this highly diffusive and radioactive gas in the detector volume. The detector was located in the Laboratoire Souterrain de Modane (LSM) at a depth of 4800 m.w.e. beneath the Frèjus mountain.

\subsection{Analysis Approach}

All NEMO-3 analyses leverage the full event reconstruction capabilities of the detector to isolate very specific event topologies. This allows very pure signal channels to be produced and also gives NEMO-3 the ability to measure and constrain its own backgrounds via various control channels. As an example, a troublesome background for $0 v \beta \beta$ searches is ${ }^{214} \mathrm{Bi}$ which beta decays, with a high Q-value, to ${ }^{214} \mathrm{Po}$ which then undergoes alpha decay with a half life of $164 \mu \mathrm{s}$. By searching for event topologies consistent with an electron followed by a delayed alpha, the activity of this isotope can be measured. This is repeated for various control channels to measure all backgrounds and the results are fitted and used to constrain the background model in the twoelectron search channel (for which a candidate event is shown in figure 2).

\section{Latest NEMO-3 Results}

The latest results of the NEMO-3 experiment are presented here for various isotopes hosted by the detector. In addition to standard $2 \gamma \beta \beta$ decay measurements and $0 v \beta \beta$ decay searches, there are also very unique physics phenomena being probed thanks to the flexibility of the NEMO approach and the long operation time of the detector.

\subsection{Results for ${ }^{100} \mathrm{Mo}$}

${ }^{100} \mathrm{Mo}$ was the most abundant isotope in the detector with approximately $6.9 \mathrm{~kg}$ of total mass. This resulted in the cleanest $2 v \beta \beta$ channel with a signal to background ratio of 76 for the phase 2 data set. The results of searching for $0 v \beta \beta$ decay with the full $34.72 \mathrm{~kg} \cdot \mathrm{yr}$ have yielded no 


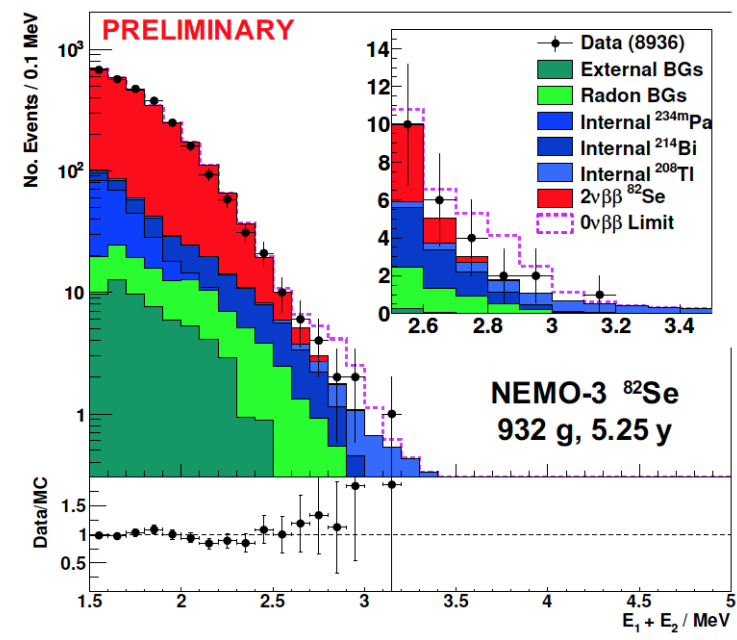

Figure 3: Distribution of the two-electron energy sum for ${ }^{82} \mathrm{Se}$ with the $0 v \beta \beta$ signal (normalized to the $90 \%$ C.L.) shown by the dashed open histogram.

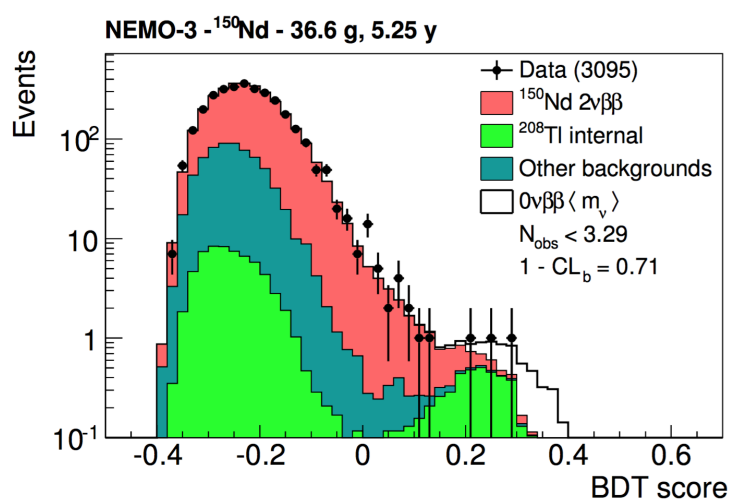

Figure 4: Output of the BDT which combines 10 detector variables into a "score" to better separate signal and background events. The open black histogram shows the $90 \%$ C.L. $0 v \beta \beta$ limit in the lightMajorana exchange hypothesis.

evidence of a signal and thus a lower limit, in the light-Majorana neutrino exchange hypothesis, for the half-life has been set at

$$
T_{1 / 2}^{0 \vee \beta \beta}>1.1 \times 10^{24} \operatorname{yr}(90 \% \text { C.L. }) .
$$

This corresponds, via equation 1.1, to a limit on the Majorana neutrino mass of $\left\langle m_{v}\right\rangle<0.3-0.6 \mathrm{eV}$ where the range is due to the aforementioned uncertainties in the NME values. Using NEMO-3's ability to distinguish between different $0 v \beta \beta$ decay modes limits have also been set on various other potential processes as summarized in [2] and [3].

\subsection{Results for ${ }^{82} \mathrm{Se}$}

The second most abundant isotope in NEMO- 3 was ${ }^{82} \mathrm{Se}$ with $0.93 \mathrm{~kg}$. Its long $T_{1 / 2}^{2 v \beta \beta}$ reduces contributions to the $0 v \beta \beta$ decay search from the irreducible $2 v \beta \beta$ decay tail. This is one of the reasons for this isotope's adoption as the baseline for SuperNEMO source foils. The preliminary results for the measurement of the $2 v \beta \beta$ half-life and the lower limit for the $0 v \beta \beta$ half-life, respectively, are

$$
\begin{aligned}
& \left.T_{1 / 2}^{2 \gamma \beta \beta}=[10.07 \pm 0.14 \text { (stat.) } \pm 0.54 \text { (syst. })\right] \times 10^{19} \mathrm{yr} \\
& T_{1 / 2}^{0 \gamma \beta \beta}>2.5 \times 10^{23} \operatorname{yr}(90 \% \text { C.L. }) .
\end{aligned}
$$

This corresponds to $\left\langle m_{v}\right\rangle<1.2-3.0 \mathrm{eV}$ which, although four times worse than the limit using ${ }^{100} \mathrm{Mo}$, was achieved with less than a $7^{\text {th }}$ of the mass. Figure 3 shows the $0 v \beta \beta$ decay search region in the two-electron sum energy distribution. 


\subsection{Results for ${ }^{116} \mathrm{Cd}$}

NEMO-3 observed $410 \mathrm{~g}$ of ${ }^{116} \mathrm{Cd}$. In addition to measuring the two-neutrino half-life, given by

$$
T_{1 / 2}^{2 v \beta \beta}=[2.74 \pm 0.04 \text { (stat.) } \pm 0.18 \text { (syst.) }] \times 10^{19} \mathrm{yr},
$$

as well as searching for $0 v \beta \beta$ decay, this analysis also focused on studying the mechanism behind $2 v \beta \beta$ decay. There are two hypotheses for how these decays occur: the single-state dominance (SSD) hypothesis predicts that the transition proceeds via the lowest-lying intermediate state while the higher-states dominance (HSD) hypothesis predicts that the higher energy states also contribute. By comparing the shapes of the single electron energy distributions it is found that SSD is slightly, but not yet conclusively, favored over HSD [4].

\subsection{Results for ${ }^{150} \mathrm{Nd}$}

With a total of $36.6 \mathrm{~g}$ in NEMO-3, ${ }^{150} \mathrm{Nd}$ is one of the most promising double-beta decay isotopes due to its high $Q_{\beta \beta}$ value of $3.4 \mathrm{MeV}$ and relatively large phase space factor. The twoneutrino half-life for this isotope has been measured to be

$$
\left.\left.T_{1 / 2}^{2 \vee \beta \beta}=[9.34 \pm 0.22 \text { (stat. })_{-0.60}^{+0.62} \text { (syst. }\right)\right] \times 10^{18} \mathrm{yr} .
$$

In the search for neutrinoless double-beta decay a multivariate approach was taken which employed the use of a Boosted Decision Tree (BDT) to improve discrimination between signal and background events. This is made possible by the full event reconstruction provided by the NEMO technique, which supplied 10 different kinematic variables into the BDT analysis (the output for which is shown in figure 4). The resultant neutrinoless half-life limit is set at

$$
T_{1 / 2}^{0 \vee \beta \beta}>2.0 \times 10^{22} \operatorname{yr}(90 \% \text { C.L. }) .
$$

This corresponds to $\left\langle m_{v}\right\rangle<1.6-5.3 \mathrm{eV}$. In using this multivariate analysis, the expected (observed) limit is $11 \%$ (34\%) better than using only the total electron energy distribution [5].

\subsection{Results for ${ }^{48} \mathrm{Ca}$}

Due to the extremely low natural abundance and the overall difficulty of enrichment there was only about $7 \mathrm{~g}$ of ${ }^{48} \mathrm{Ca}$ in NEMO-3. It is however, a highly desirable isotope to study since it has the largest $Q_{\beta \beta}$ value, $4.3 \mathrm{MeV}$, which is well above almost all background processes. The measured $2 v \beta \beta$ half-life for this isotope was measured to be

$$
\left.\left.T_{1 / 2}^{2 \vee \beta \beta}=\left[6.4_{-0.6}^{+0.7} \text { (stat. }\right)_{-0.9}^{+1.2} \text { (syst. }\right)\right] \times 10^{19} \text { yr. }
$$

This represents the most precise measurement, to date, for this isotope [6].

\subsection{Other Analyses}

The NEMO technique also lends itself to studying other physics phenomena beyond doublebeta decays. Two such analyses are briefly mentioned here. The first of these is a search for neutrinoless quadruple-beta decay $(0 \vee 4 \beta)$. This process, which also violates lepton number, postulates 
that neutrino's are strictly Dirac particles and thus forbids $0 v \beta \beta$ decay [7]. The best isotope, out of only three possible candidates, is considered to be ${ }^{150} \mathrm{Nd}$. Thanks to its ability to fully reconstruct event topologies and discriminate those with a four-electron final state NEMO-3 is well suited to search for such a decay.

The second unique physics analysis being performed is the search for periodically varying nuclear decay rates. Recent claims for potentially unexplained annual modulations such as in [8] and [9] have motivated the first search for periodicities in double-beta decay rates, specifically in ${ }^{100} \mathrm{Mo}$. The Lomb-Scargle periodogram technique is being used to search for any such modulations, ranging from daily to multi-year effects, in the low-radon data set spanning just over six years.

\section{Conclusions}

The power and versatility of NEMO-3's tracking-calorimetry technique is made evident in the breadth of $2 v \beta \beta$ decay measurements and $0 v \beta \beta$ searches and the fact that the results were obtained using such small isotopic masses also speaks to its capabilities in providing a very low, and potentially background free, technique. In addition to all of the physics measurements made and still to come from studying the six isotopes present in the detector, NEMO-3 also continues to provide interesting and unique physics processes beyond double-beta decay.

\section{Acknowledgements}

The NEMO-3 and SuperNEMO collaborations would like to thank the staff of the LSM laboratory. We also acknowledge support by the grants agencies of the Czech Republic, CNRS/IN2P3 in France, RFBR in Russia, STFC in the U.K. and NSF in the U.S.

\section{References}

[1] J. Schechter and J. W. F. Valle, Phys. Rev. D 25 (1982) 2951.

[2] R. Arnold et al., Phys. Rev. D 89 (2014) 111101.

[3] R. Arnold et al., Phys. Rev. D 92 (2015) 072011.

[4] R. Arnold et al. arXiv:1610.03226 [hep-ex].

[5] R. Arnold et al., Phys. Rev. D 94 (2016) 072003.

[6] R. Arnold et al., Phys. Rev. D 93 (2016) 112008.

[7] J. Heeck and W. Rodejohann, Europhys. Lett. 103 (2013) 32001.

[8] J. H. Jenkins, et al., Astropart. Phys. 32 (2009) 42.

[9] R. Bernabei, et al., Eur. Phys. J. C 73 (2013) 2648. 